\title{
Força de preensão palmar: métodos de avaliação e fatores que influenciam a medida
}

\author{
Hand grip strength: evaluation methods and factors influencing \\ this measure
}

Jonathan Ache Dias Angélica Cristiane Ovando ${ }^{2}$ Wladymir Külkamp Noé Gomes Borges Junior ${ }^{1}$

\begin{abstract}
1 Universidade do Estado de Santa Catarina. Centro de Ciências da Saúde e do Esporte. Laboratório de Instrumentação. Florianópolis, SC. Brasil.
\end{abstract}

2 Universidade do Estado de Santa Catarina. Centro de Ciências da Saúde e do Esporte. Laboratório de Control Motor. Florianópolis, SC. Brasil.

3 Universidade do Estado de Santa Catarina. Centro de Ciências da Sáude e do Esporte.. Laboratório Multisetorial.Florianópolis, SC. Brasil.

Recebido em 22/06/09 Revisado em 23/10/09 Aprovado em 30/11/09
Resumo - A avaliação da força de preensão manual (FPM) do tipo palmar é frequentemente utilizada, especialmente, no âmbito fisioterápico e desportivo. A busca por valores normativos tem sido foco de alguns estudos, mas os diferentes instrumentos e protocolos dificultam a generalização dos resultados. Além disso, existem diversos fatores que podem influenciar a FPM como o sexo, a idade, a dominância, o horário de avaliação, o posicionamento corporal, a sinceridade do esforço, as características antropométricas e o tamanho da empunhadura. Este trabalho teve como objetivo realizar uma revisão crítico-narrativa do estado da arte relacionado a medidas da FPM e alguns aspectos referentes à sua interpretação. De acordo com o universo bibliográfico revisado, em avaliações de FPM parece ser fundamental a utilização de um dinamômetro que permita a mensuração de curvas força vs tempo e que possibilite o ajuste contínuo da empunhadura de acordo com as dimensões da mão. Recomenda-se ainda: padronizar o horário de avaliação, avaliar ambas as mãos, realizar no mínimo três avaliações em cada mão, adotar um posicionamento corporal padrão, utilizar incentivo verbal e/ou visual e corrigir a força pela massa corporal. A padronização dos métodos de avaliação e interpretação dos resultados pode permitir a construção de valores de referência confiáveis e universalmente aceitos.

Palavras-chave: Força da Mão; Biomecânica; Antropometria.

Abstract - The evaluation of hand grip strength (HGS) is commonly used, especially in physiotherapy and sports. The establishment of reference values has been the focus of some studies, but the different instruments and protocols used impair generalization of the results. Moreover, several factors influence HGS, such as gender, age, dominance, time of assessment, body position, sincerity of effort, anthropometric characteristics, and grip span. The objective of this study was to review the current state of the art regarding HGS measures and some aspects related to their interpretation. According to the literature, the use of a dynamometer that allows the construction of force vs. time curves and the continuous adjustment of grip span according to hand dimensions seems to be essential for the evaluation of HGS. Additional recommendations are the standardization of the time of evaluation, assessment of both hands, a minimum number of three trials for each hand, adoption of a standard body position, use of verbal and/ or visual encouragement, and the correction of muscle strength according to body weight. The standardization of evaluation methods and interpretation of the results permits the establishment of reliable and universally accepted reference values of HGS.

Key words: Hand Strength; Biomechanics; Anthropometry. 


\section{INTRODUÇÃO}

A mão é considerada um dos principais instrumentos do corpo humano, sendo que boa parte do desenvolvimento da humanidade pode ser creditado a ela, especialmente, pela peculiar característica de possibilitar movimentos de preensão. Existem dois tipos básicos de preensão amplamente apresentados na literatura: a de força, que consiste na ação de flexão dos dedos sobre a região palmar, e a de precisão, relacionada à aproximação dos dedos polegar e indicador.

Habitualmente, a avaliação da força de preensão manual (FPM) é utilizada como parâmetro na prática clinica, desempenhando um papel importante no controle de processos de reabilitação ${ }^{1}$, na avaliação e tratamento de desordens músculoesqueléticas da mão e na avaliação de pessoas com patologias neurológicas ${ }^{3}$. Além disso, a FPM é entendida como indicador geral de força e potência musculares ${ }^{4}$, podendo ser relacionada a taxas de mortalidade ${ }^{5}$. É útil, ainda, na área esportiva ${ }^{6-8}$, na reabilitação ocupacional ${ }^{2}$, em testes de admissão em diversos tipos de trabalho e na ergonomia?

Alguns estudos ${ }^{10-12}$ buscaram estabelecer valores normativos do desempenho da FPM, no entanto, os diferentes protocolos de coleta e instrumentos utilizados dificultam a generalização dos resultados. Além disso, outros fatores podem dificultar o estabelecimento de valores normativos universalmente aceitos. Entre eles podem ser citados: sexo, idade, dominância ${ }^{13}$, horário de avaliação ${ }^{14}$, posicionamento corporal ${ }^{9,15}$ e características antropométricas $^{16,17}$. Adicionalmente e não menos importante, o ajuste do tamanho da empunhadura utilizada no dinamômetro de preensão manual também é largamente debatido na literatura ${ }^{11,18-21}$, de maneira que parece haver a necessidade de ajustar o equipamento para diferentes tamanhos de mão.

Apesar de a literatura especializada estar repleta de pesquisas que utilizam a FPM como objeto principal de estudo, existe certa carência de trabalhos que se proponham a reunir e detalhar as características concernentes à avaliação dessa variável, especialmente, no que diz respeito aos fatores intervenientes na medida e à falta de padronização dos métodos.

Deste modo, este trabalho teve como objetivo realizar uma revisão crítico-narrativa do estado da arte referente à medida da FPM do tipo palmar, suas características, métodos de avaliação, fatores que influenciam a medida e o ajuste do tamanho da empunhadura, analisando de maneira crítica as informações disponíveis na literatura. Isto pode ser especialmente proveitoso para pesquisadores, médicos e fisioterapeutas que trabalham com a medida da preensão manual, bem como para pesquisadores da área desportiva, atletas e técnicos que utilizam esse tipo de avaliação como ferramenta essencial de trabalho.

Os artigos desta revisão foram selecionados a partir das bases de dados Science Direct, PubMed, SpringerLink, LILACS, Informaworld e IngentaConnect, no período entre março de 2008 e abril de 2009. Foram incluídos nesta revisão apenas os artigos publicados na íntegra, entre 1980 e 2009, que apresentavam como descritores os termos handgrip, handgrip strength, grip strengh e força de preensão manual ou palmar.

\section{MÉTODOS DE AVALIAÇÃO DA PREENSÃO MANUAL}

A FPM tem sido investigada, principalmente, por meio da medição da força isométrica máxima que pode ser exercida sobre um dinamômetro, em inúmeros padrões de empunhadura ou pegada. A preensão power grip, conhecida como palmar e a mais referenciada na literatura, origina-se do termo grasp, que significa apertar um objeto cilíndrico. Nesse tipo de pegada existe a inibição da ação do polegar, como apresentado na figura 1 .

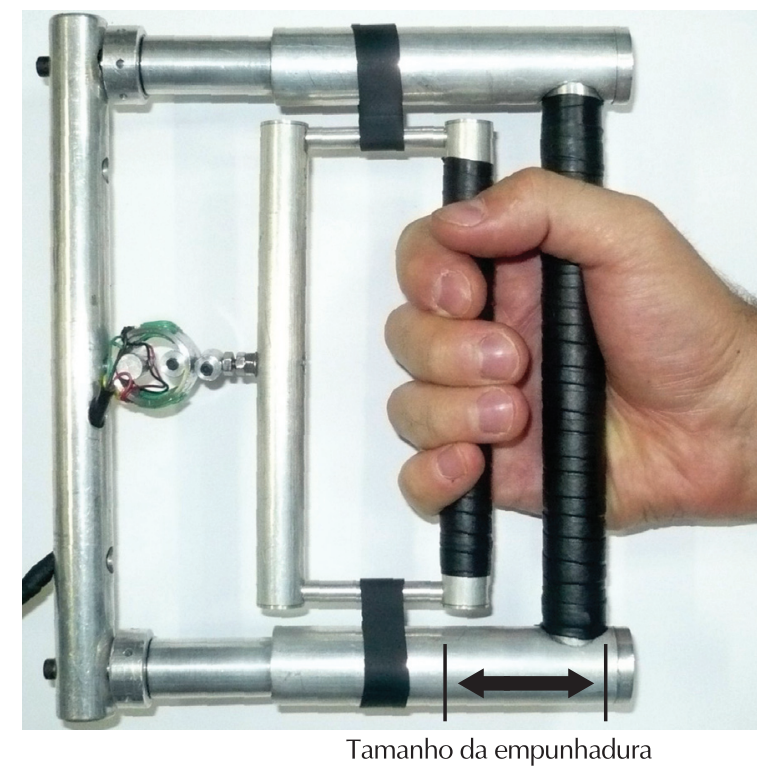

Figura 1. Preensão palmar em um dinamômetro.

Dinamômetros são equipamentos que permitem a mensuração da força aplicada em um sistema baseado em células de carga. São divididos nos tipos isométrico e isocinético, sendo que para medidas de FPM, tradicionalmente, têm sido usados dinamômetros 
isométricos, de característica analógica ou digital.

Dentre os diversos equipamentos encontrados hoje no mercado, o dinamômetro hidráulico analógico Jamar ${ }^{\circledR}$ (Lafayette Instrument, EUA) é largamente utilizado, sendo considerado um equipamento padrão ouro ${ }^{22}$. No entanto, esse equipamento, assim como o Digital Hand Dynamometer (T.K.K. 5401 Grip-D, Takey ${ }^{\circledR}$, Smedley; Tokyo, Japan), são capazes de detectar somente o valor de pico de força máxima alcançado (Fmax). Outros dinamômetros, do tipo extensiométrico como o Qubit (Qubit Systems Inc., Kingston, Canada) e o dinamômetro desenvolvido pelo laboratório de Instrumentação da UDESC (figura 1), por exemplo, são instrumentos que permitem adquirir valores de força em unidade de tempo (curva força vs tempo).

Diferentes métodos têm sido utilizados para avaliar a FPM, sendo que as diferenças estão relacionadas à intensidade da contração (máxima ou sub-máxima), ao tempo de duração da contração e ao número de repetições realizadas (contínuas ou intermitentes).

A maioria dos trabalhos encontrados na literatura avalia o pico de força alcançado em um intervalo de três a 10 segundos, bem como o tempo necessário para sustentar $50 \%$ da Fmax ${ }^{16,17,21,23}$. 25. Valores normativos da FPM medida por esse método têm sido sugeridos e são apresentados, por exemplo, nos trabalhos de Luna-Heredia, Martín-Peña e Ruiz-Galiana ${ }^{10}$, Bohannon et al. ${ }^{25}$ e Günther et al. ${ }^{26}$.

Embora valores de Fmax possam ser úteis na praxis clínica, desportiva e até mesmo científica, existe um amplo espectro de variáveis que ocorrem entre o início e o final da preensão que podem auxiliar na melhor compreensão do fenômeno. Isso pode ser especialmente válido, por exemplo, em estudos do processo de fadiga da musculatura envolvida na FPM ${ }^{13,27-32}$. Por esse motivo, geralmente, as curvas de FPM são analisadas com protocolos que variam entre cinco segundos e seis minutos de contração máxima ou sub-máxima.

A análise e interpretação da curva de preensão (figura 2) não são recentes. Em 1980, Myers, Grennan e Palmer ${ }^{27}$, já estabeleciam como critério o uso de parâmetros como: pico de força máxima (Fmax), taxa de desenvolvimento de força (TDF), taxa de perda de força $(\% \mathrm{~F})$ entre a Fmax e a força final (Ffinal) e o trabalho realizado (Impulso). Esses parâmetros continuam a ser utilizados até os dias de hoje e podem ser melhor compreendidos, observando-se a figura 2 . A variável que representa o trabalho realizado, neste caso, é o impulso, defi- nido pela integração da área formada pela curva. Byrd e Jenness ${ }^{28}$ sugerem que a área abaixo da curva de preensão pode ser uma ferramenta alternativa para estudos que envolvem resistência muscular. Já Ikemoto et al. ${ }^{4}$, utilizando um protocolo de cinco segundos, sugerem a utilização de um método de integração da área abaixo da curva da FPM para estimar potência muscular.

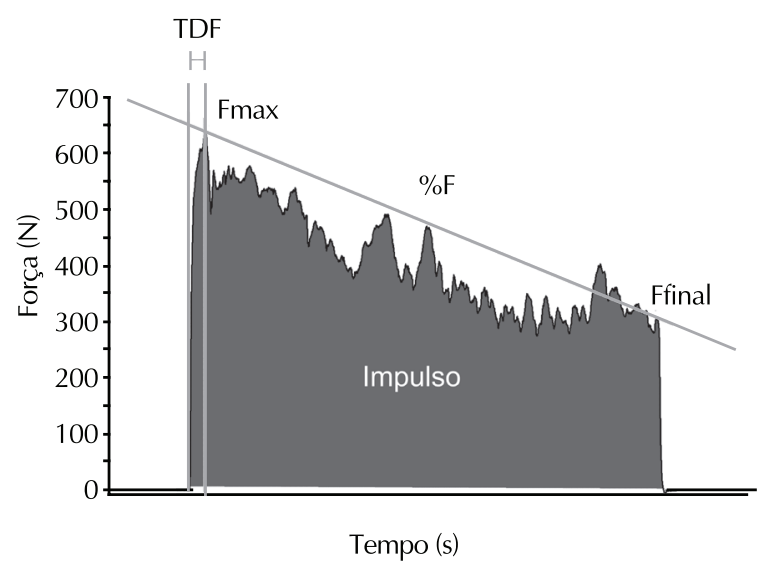

Figura 2. Parâmetros da curva de força de preensão manual geralmente avaliados.

Yamaji et al. ${ }^{30}$ verificaram o efeito de diferentes percentuais de Fmax na realização de testes de FPM em protocolos de um, três e seis minutos. Em um protocolo de força máxima, o comportamento da curva pôde ser explicado por uma função exponencial. Demonstraram, também, que a $75 \%$ e $50 \%$ da Fmax, ao contrário, o comportamento da curva é diferente, perdendo a característica exponencial. Por este motivo, segundo os autores, pode não ser possível a avaliação da fadiga da força de preensão durante um minuto a $50 \%$ da Fmax, já que a força nesse ponto da curva pode ainda não apresentar declínio. Sugerem, ainda, que em testes prolongados (mais de três minutos) o desempenho da força de preensão é afetado por fatores sensoriais intervenientes como a dor.

Apesar da busca de alguns pesquisadores por valores de referência e da prática comum de comparações entre diferentes estudos, muitos trabalhos pecam na escolha dos instrumentos e no posicionamento dos avaliados ${ }^{25}$. A Sociedade Americana de Terapeutas da Mão (ASHT) recomenda a utilização do dinamômetro Jamar ${ }^{\circledR}$, na segunda posição (ele possui cinco), referente ao tamanho da empunhadura. Os sujeitos devem permanecer sentados em uma cadeira tipo escritório (sem braços) com a coluna ereta, mantendo o ângulo de flexão do joelho em $90^{\circ}$, o ombro posicionado em adução e rotação neutra, o cotovelo flexionado a $90^{\circ}$, com 
antebraço em meia pronação e punho neutro, podendo movimentá-lo até $30^{\circ}$ graus de extensão. $\mathrm{O}$ braço deve ser mantido suspenso no ar com a mão posicionada no dinamômetro, que é sustentado pelo avaliador ${ }^{22}$. A negligência com relação ao uso de uma padronização universal, levando em conta os aspectos abordados neste parágrafo, que têm efeito direto sobre o desempenho da preensão manual, torna muito difícil o desenvolvimento de trabalhos cujos resultados possam ser agrupados e/ ou comparados entre diferentes populações.

Ainda com relação à necessidade de padronização, a FPM pode ser avaliada de três maneiras: em tentativa única, adotando-se uma média das tentativas ou ainda assumindo o melhor desempenho dentre duas ou três tentativas. Haidar et al. ${ }^{23}$ compararam a realização de uma tentativa e a média de três tentativas, observando altos índices de concordância entre ambos os protocolos. Todavia, os autores relataram que, aproximadamente, 25\% dos avaliados obtiveram maiores valores de força máxima na segunda ou terceira tentativa. Portanto, parece ser fundamental para comparação dos resultados de diferentes estudos, que os mesmos tenham adotado critérios metodológicos semelhantes em relação ao número de tentativas.

O último aspecto, a ser aqui abordado, relacionado aos métodos de avaliação da FPM diz respeito à utilização do incentivo ao sujeito avaliado. Como em todo teste de esforço máximo, a utilização do incentivo verbal ou visual pode influenciar significativamente os resultados. No caso da FPM, não há um consenso entre os pesquisadores da área, de maneira que muitos utilizam o incentivo verbal ${ }^{3} \mathrm{e}$ outros o incentivo com feedback visual de desempenho $^{30}$. Sugere-se aos pesquisadores que adotem o mesmo tipo de incentivo utilizado nos estudos que pretendem comparar, a fim de garantir que essa variável não interfira na interpretação dos resultados.

\section{FATORES QUE INFLUENCIAM A MEDIDA DA FORÇA DE PREENSÃO}

Dentre os aspectos que influenciam a medida da FPM está o horário de avaliação. No estudo de Young et al. ${ }^{33}$ foi testada a flutuabilidade da força entre os períodos da manhã e da tarde. Não foram encontradas diferenças significativas após 12 semanas de avaliação. Todavia, a força de preensão palmar variou em média 50,0 e 82,4 N no período da manhã e da tarde, respectivamente.

McGarvey et al. ${ }^{14}$ encontraram diferenças significativas (5 a $7 \%$ ) da força de pico entre diferentes horários, em um mesmo dia. Apesar de os valores encontrados serem aparentemente pequenos para sugerir cautela em estudos clínicos, os autores sugerem a padronização do horário para evitar interferências.

Outro fator que parece ser interveniente na medida está relacionado à dominância lateral. Nicolay e Walker ${ }^{13}$ avaliaram 51 sujeitos saudáveis, com idade entre 18 e 33 anos. Seus resultados mostraram que, em relação ao pico de força máxima, em mulheres a mão dominante possui geralmente melhor desempenho. Os homens, embora não tenham apresentado diferenças de força entre as mãos, parecem ter valores mais altos de força que as mulheres. Os autores relataram, adicionalmente, que a mão dominante, apesar de gerar mais força, apresenta fadiga mais rapidamente, independente do sexo.

O efeito da dominância também foi investigado por Armstrong e Oldham ${ }^{34}$. Os mesmos concluíram que as diferenças encontradas entre a FPM da mão dominante e não-dominante de 83 pessoas, com idade entre 18 e 72 anos, são pequenas (em torno de $10 \%$ ) e que a utilização desses resultados deve ser adotada com cautela na elaboração de delineamentos metodológicos de intervenção clínica ou de pesquisa.

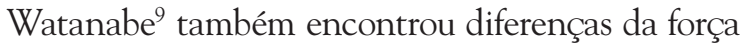
em torno de $10 \%$ entre as mãos, em um grupo de 50 mulheres e 50 homens da mesma faixa etária do estudo anterior. Nesse estudo, os homens realizaram FPM de 443,2 \pm 68,6 N na mão dominante e 415,8 \pm $57,8 \mathrm{~N}$ não mão não-dominante e as mulheres 278,5 $\pm 41,1 \mathrm{~N}$ e $259,9 \pm 34,3 \mathrm{~N}$, respectivamente.

De modo geral, a mão dominante apresenta melhor desempenho que a mão não-dominante com relação à Fmax, em ambos os sexos ${ }^{10,12}$.

Variáveis antropométricas também podem configurar-se como fatores influenciadores das medidas. Valores normativos de FPM têm sido encontrados e relacionados com a massa corporal, estatura, perímetro do antebraço e tamanho da mão. As medidas ou proporções corporais têm sido utilizadas para predizer a FPM, auxiliando na avaliação clínica da perda funcional de membros superiores, principalmente, a mão, em caso de doença ou acidente e na seleção de atletas ${ }^{35}$. Ou seja, a partir de características antropométricas pode ser possível predizer o valor de FPM de uma pessoa.

Mitsionis et al. ${ }^{12}$ encontraram em seu estudo com 232 adultos, associação positiva entre a estatura e a FPM em ambos os sexos, associação positiva com a massa corporal somente nos homens e associação positiva entre índice de massa corporal (IMC) e a FPM somente nas mulheres. Jürimäe, Hurbob e Jüri- 
mäe ${ }^{36}$ encontraram $76,1 \%$ de influência da estatura sobre a FPM em meninos, entre oito e 11 anos de idade. Os autores concluíram que fatores como estatura, perímetro do antebraço, massa gorda e óssea influenciam o desempenho da FPM. No trabalho de Luna-Heredia, Martín-Peña e Ruiz-Galiana ${ }^{10}$, também foi encontrada relação entre a estatura e a FPM, além de serem estabelecidos valores de referência.

Neu et al. ${ }^{16}$ relacionaram a FPM com a área de secção transversa dos músculos do antebraço em adultos e adolescentes. Os autores observaram que a FPM, quando corrigida pela área de secção transversa dos músculos do antebraço, aumentou em metade dos avaliados com idade entre seis e 20 anos. Todavia, Bertuzzi, Franchini e Kiss ${ }^{6}$ encontraram, em um grupo de escaladores elite, uma correlação moderada $(0,740)$ entre o perímetro do antebraço e a Fmax.

Nicolay e Walker ${ }^{13}$ encontraram alta relação da Fmax com o tamanho e perímetro do antebraço, perímetro do punho, a largura da palma da mão, o tamanho da palma da mão, a massa corporal e a estatura. Altas relações também foram encontradas entre as mesmas variáveis antropométricas e a média de força aplicada durante 30 segundos de pressão contínua.

Outros trabalhos analisaram mais especificamente os efeitos das dimensões da mão sobre o desempenho da FPM. Visnapuu e Jurimae ${ }^{37}$ encontraram influência do comprimento do dedo médio e do perímetro da mão na FPM, em atletas de basquetebol, ao contrário de Nicolay e Walker ${ }^{13}$ que não encontraram associação entre essas variáveis. Clerke, Clerke e Adams ${ }^{17}$, analisando 228 adolescentes, não encontraram efeito das formas da mão (quadrada, intermediária e longa) sobre a capacidade de gerar FPM máxima.

A massa corporal também parece exercer influência na interpretação das medidas de FPMํ. Em relação à utilização de normalização ou correção da FPM, alguns autores ${ }^{38,39}$ sugerem a utilização de uma escala alométrica para ajustar os valores de força máxima pela massa corporal ou massa magra, a fim de permitir comparações entre diferentes indivíduos, bem como para estabelecer valores normativos universalmente aceitos. A equação sugerida pode ser descrita da seguinte forma:

Força Corrigida $=$ Fmax $* \mathrm{MC}^{\mathrm{b}}$

onde MC é a massa corporal e "b" é o expoente alométrico de ajuste.

Vanderburgh et al..$^{38} \mathrm{em}$ seu estudo, desenvolvido a partir de testes com 279 estudantes americanos e ingleses, encontraram um expoente alométrico (b) igual a 0,51 (equação 1), permitindo o ajuste da FPM pela MC em ambos os sexos.

Markovic e Jaric ${ }^{39}$, utilizando 77 estudantes (18 aos 26 anos) e analisando avaliações de força de outros grupos musculares, além dos responsáveis pela preensão manual, sugerem a utilização do expoente 0,67 . Pua $^{40}$ ao avaliar 131 idosos, sugeriu um expoente de 0,63.

O posicionamento dos segmentos corporais também parece influenciar o desempenho da FPM. Su et al..$^{15}$ utilizaram em seu estudo 80 homens e 80 mulheres chinesas e adotaram como base o protocolo sugerido pela ASHT, apresentado no capítulo anterior desta revisão. Os maiores valores de FPM foram encontrados quando o sujeito estava com o ombro em flexão de $180^{\circ}$ e o cotovelo estendido. Os menores valores foram encontrados quando o ombro estava neutro e o cotovelo flexionado $90^{\circ}$ como o sugerido pela ASHT. Mais recentemente, Watabane et al. ${ }^{9}$ investigando o efeito da postura sobre a FPM, não encontraram diferenças entre os valores de força máxima entre a posição sentada e em pé. A posição deitada apresentou os menores resultados.

Com relação à interferência na medida, outro aspecto abordado na literatura diz respeito à sinceridade do esforço do avaliado. A diferença entre o pico e a média da força exercida em um dinamômetro, durante cinco segundos ${ }^{29}$ e os ângulos de inclinação e declinação da curva de FPM ${ }^{31}$ parecem ser bons indicadores da sinceridade de esforço por parte do avaliado. No entanto, para Tredgett e Davis ${ }^{1}$ testes de preensão de curta duração (cinco a 10 segundos) podem não ser bons indicadores de sinceridade de esforço.

De qualquer maneira, a sinceridade do esforço pode depender de vários aspectos como: o psicológico (motivação), o próprio entendimento do avaliado em relação ao significado do teste, a dor (pelo desconforto do dinamômetro que esta sendo utilizado), entre outros. Recomenda-se que o avaliado esteja ambientado com o dinamômetro, que o mesmo compreenda o objetivo do teste e que seja utilizado incentivo verbal e/ou visual.

\section{TAMANHO DA EMPUNHADURA}

As dimensões da mão e o tamanho da empunhadura também podem influenciar o desempenho da FPM. O tamanho da empunhadura é a distância entre o apoio da palma da mão e dos dedos quando mensuramos a FPM com a utilização de um dinamômetro (figura 1). Essa distância é fixa em 
alguns instrumentos, discreta em outros (regulagem restrita da amplitude) e com ajuste contínuo somente em um número limitado de equipamentos (figura 1), tornando-se possível ajustá-los a qualquer tamanho de mão.

Quando se utiliza o dinamômetro Jamar®, o ajuste da empunhadura utilizado não varia muito de estudo para estudo, sendo geralmente definido como posição dois e três (130 e 160 mm de circunferência), ao contrário de estudos que utilizam dinamômetros com ajuste contínuo. Blackwell et al. ${ }^{18}$ analisaram o efeito do tamanho da empunhadura sobre o desempenho da FPM (submáxima - 60 a 65\% da Fmax) em 18 homens saudáveis. Utilizando um dinamômetro Jamar® o estudo detectou que o tamanho intermediário de empunhadura (posições dois e três) permitiu que a população estudada obtivesse os melhores resultados. Entretanto, Boadella et al..$^{24}$ afirmam que quando se utiliza um dinamômetro com ajuste discreto do tamanho da empunhadura, a autosseleção do tamanho da empunhadura por parte do avaliado promove melhor desempenho.

Ruiz-Ruiz et al. ${ }^{19}$ propõem a utilização da empunhadura de $55 \mathrm{~mm}$ fixa para homens adultos. Para as mulheres, o autor sugere uma equação baseada no tamanho da mão quando forem utilizados dinamômetros que dispõem do ajuste contínuo da empunhadura:

$y=x / 5+15 \mathrm{~mm}$

onde " $y$ " é a empunhadura e " $x$ " o tamanho da mão, chamado na literatura de Hand Span (distância entre a extremidade do dedo mínimo e extremidade do polegar quando a mão está aberta o máximo possível).

Em outro estudo ${ }^{21}$ os autores sugerem a utilização de equações baseadas no tamanho da mão para fazer o ajuste de empunhadura de adolescentes de 13 a 18 anos. As equações apresentadas para o melhor ajuste do tamanho da empunhadura para meninos e meninas foram, respectivamente, as equações 3 e 4 .

$$
\begin{aligned}
& y=x / 7.2+3.1 \mathrm{~cm} \\
& y=x / 4+1.1 \mathrm{~cm}
\end{aligned}
$$

Seguindo os mesmos métodos, España-Romero et al. ${ }^{11}$ sugeriram, recentemente, a utilização de outras equações para meninos e meninas de seis a 12 anos (equações 5 e 6 respectivamente)

$$
y=(x / 4)+0.44
$$

$$
y=0.3 x+0,52
$$

Por outro lado, outros autores sugerem utilizar outras medidas da mão para definir o tamanho da empunhadura. Segundo Watanabe et al. ${ }^{9}$, a utilização do tamanho da empunhadura, assumindo a metade da distância entre a extremidade da falange distal do dedo indicador e a junção metacarpofalangeana do mesmo, parece ser a ideal. Em seu estudo, foram analisados 100 sujeitos entre homens e mulheres, avaliados com um dinamômetro Takei. Os resultados demonstraram que o tamanho da empunhadura ajustado pelo tamanho do dedo indicador ou este com um acréscimo de $10 \%$ parece ser o tamanho ideal de empunhadura para ambos os sexos.

Eksioglu ${ }^{20}$ propõe a utilização de outro parâmetro para o ajuste do melhor tamanho da empunhadura. A medida $\mathrm{TCL}_{\mathrm{m}}$, que foi definida pelo autor como a distância entre a extremidade da falange proximal do dedo médio até a base do polegar, é inspirada na posição que é adotada durante a execução da FPM, ilustrada na figura 1. Em seu estudo, 12 pessoas executaram a FPM com nove diferentes tamanhos de empunhadura, sendo que a subtração de $2,5 \mathrm{~cm}$ da medida $\mathrm{TCL}_{\mathrm{m}}\left(\mathrm{TCL}_{\mathrm{m}}-2,5\right)$ apresentou os melhores resultados.

Dessa forma, a escolha do parâmetro antropométrico utilizado no ajuste do tamanho ideal de empunhadura parece ser de extrema importância. Recomenda-se cautela, todavia, na escolha desse parâmetro, visto que ainda não existe um consenso entre pesquisadores, apesar de o parâmetro tamanho da mão estar sendo bastante utilizado na literatura especializada.

De modo geral, quando for utilizado um dinamômetro que permite ajuste contínuo da empunhadura, recomenda-se a utilização das equaÇões propostas por Ruiz-Ruiz et al. ${ }^{19}$ quando forem avaliados adultos e de Ruiz-Ruiz et $\mathrm{al}^{21}$ e EspañaRomero et al. ${ }^{11}$ quando forem crianças e adolescentes. Já quando forem utilizados dinamômetros com ajuste discreto da empunhadura, como o Jamar®, parece que a posição autossugerida permite melhor desempenho.

\section{CONSIDERAÇÕES FINAIS}

Com base na literatura revisada, pode ser observado que dinamômetros extensiométricos que permitam a aquisição da curvas força vs tempo podem fornecer uma gama maior de variáveis para análise, em comparação a dinamômetros que permitem apenas 
a medida de Fmax. Adicionalmente, a escolha por instrumentos que permitam um ajuste contínuo da empunhadura parece ser mais sensata, haja vista o efeito que essa variável parece exercer sobre o desempenho de FPM.

Sugere-se que alguns procedimentos básicos sejam adotados em qualquer investigação que envolva medidas de FPM, são eles: a padronização do horário de avaliação; a avaliação de ambas as mãos devido ao efeito da dominância; a realização de no mínimo três avaliações em cada mão; a adoção de um posicionamento corporal padrão, de preferência o sugerido pela ASHT; o ajuste do tamanho da empunhadura, levando em consideração o sexo e o tamanho da mão e finalmente, a utilização de incentivo verbal e/ou visual na tentativa de garantir esforço máximo durante a tarefa.

Cabe ainda referir que o uso de métodos de correção da força pela $\mathrm{MC}$, tais como equações alométricas, podem ser úteis na determinação de valores normativos generalizados e na comparação de diferentes indivíduos.

\section{Agradecimentos}

$\mathrm{O}$ primeiro e segundo autores agradem a CAPES pela bolsa de mestrado.

\section{REFERÊNCIAS BIBLIOGRÁFICAS}

1. Tredgett MW, Davis TRC. Rapid repeat testing of grip strength for detection of faked hand weakness. J Hand Surg [Br] 2000;25(4):372-375.

2. Sande LP, Coury HJCG, Oishi J, Kumar S. Effect of muscoloskeletal disorders on prehension strength. Appl Ergon 2001;32(6):609-616.

3. Meldrum D, Cahalane E, Conroy R, Guthrie R, Hardiman O. Quantitative assessment of motor fatigue: normative values and comparison with prior-polio patients. Amyotroph Lateral Scler 2007;8(3):170-176.

4. Ikemoto Y, Demura S, Yamaji S, Minami M, Nakada M, Uchiyama M. Force-time parameters during explosive isometric grip correlate with muscle power. Sport Sci Health 2007;2(2):64-70.

5. Rantanen T, Volpato S, Ferrucci L, Heikkinen E, Fried LP, Guralnik, JM. Handgrip strength and cause-specific and total mortality in older disabled women: exploring the mechanism. J Am Geriatr Soc 2003;51(5):636-641.

6. Bertuzzi RCM, Franchini E, Kiss MAPD. Análise da força e da resistência de preensão manual e as suas relações com variáveis antropométricas em escaladores esportivos. Rev Bras Ciên Mov 2005;13(1):87-93.

7. Leyk D, Gorges W, Ridder D, Wunderlich M, Rüther T, Sievert A, et al . Hand-grip strength of young men, women and highly trained female athletes. Eur J Appl Physiol 2007;99(4):415-421.

8. Borges Junior N, Domenech SC, Silva ACK, Dias JA, Sagawa Junior Y. Estudo comparativo da força de preensão isométrica máxima em diferentes modalidades esportivas. Rev Bras Cineantropom Desempenho Hum 2009;11(3):292-298.

9. Watanabe T, Owashi K, Kanauchi Y, Mura N, Takahara M, Ogino T. The short-term reliability of grip strength measurement and the effects of posture and grip span. J Hand Surg 2004;30(3):603-609.

10. Luna-Heredia E, Martín-Peña G, Ruiz-Galiana, J. Handgrip dynamometry in healthy adults. Clin Nutr 2005;24(2):250-258.

11. España-Romero V, Artero EG, Santaliestra-Pasias AM, Gutierrez A, Castillo MJ, Ruiz-Ruiz, J. Hand Span Influences Optimal Grip Span in Boys and Girls Aged 6 to 12 Years. J Hand Surg 2008;33(3):378-384.

12. Mitsionis G, Pakos EE, Stafilas KS, Paschos N, Papakostas T, Beris AE. Normative data on hand grip strength in a Greek adult population. Int Orthop 2009;33(3): 713-717.

13. Nicolay CW, Walker AL. Grip strength and endurance: Influences of anthropometric variation, hand dominance, and gender. Int J Ind Ergonom 2005;35(7):605-618.

14. McGarvey SR, Morrey BF, Askew LJ, An KN. Reliability of isometric strength testing: temporal factors and strength variation. Clin Orthop Relat Res 1984;185(1):301-305

15. Su, C-Y; Lin, J-H; Chien, T-H; Cheng, K-F; Sung, Y-T. Grip strength in different positions of the elbow and shoulder. Am J Phys Med Rehabil 1994;75(7):812-815.

16. Neu CM, Rauch F, Rittweger J, Manz F, Schoenau E. Influence of puberty on muscle development at the forearm. Am J Physiol Endocrinol Metab 2002;46(1):103-107.

17. Clerke AM, Clerke JP, Adams RD. Effects of hand shape on maximal isometric grip strength and its reliability in teenagers. J Hand Ther 2005;18(1):19-29.

18. Blackwell JR, Kornatz KW, Heath EM. Effect of grip span on maximal grip force and fatigue of flexor digitorum superficialis. Appl Ergon 1999;30(5):401-405.

19. Ruiz-Ruiz J, Mesa JLM, Gutiérrez A, Castillo MJ. Hand size influences optimal grip span in women but not in men. J Hand Surg 2002;27(5):897-901.

20. Eksioglu M. Relative optimum grip span as a function of hand anthropometry. Int J Ind Ergon 2004;34(1):1-12

21. Ruiz-Ruiz J, Espana-Romero V, Ortega FB, Sjöström M, Castillo MJ, Gutierrez A. Hand Span Influences Optimal Grip Span in Male and Female Teenagers. J Hand Surg 2006;31(8):1367-1372.

22. Fess EE. Grip strength. In: Casanova JS, editor. Clinical assessment recommendations. 2nd ed. Chicago: American Society of Hand Therapists; 1992. p. 41-45.

23. Haidar SG, Kumar D, Bassi RS, Deshmukh SC. Average versus maximum grip strengh: Which is more consistent? J Hand Surg 2004;29(1):82-84.

24. Boadella JM, Kuijer PP, Sluiter JK, Frings-Dresen $\mathrm{MH}$. Effect of self-selected handgrip position on maximal handgrip strength. Arch Phys Med Rehabil 2005;86(2):328-331.

25. Bohannon RW, Peolsson A, Massy-Westropp N, Desrosiers J, Bear-Lehman J. Reference values for adult grip strength measured with a Jamar dynamometer: a descriptive metaanalysis. Physiotherapy 2006;92(1):11-15.

26. Günther CM, Bürger A, Rickert M, Crispin A, Schulz CU. Grip Strength in Healthy Caucasian Adults: Reference Values. J Hand Surg 2008;33(4):558-565. 
27. Myers DB, Grennan DM, Palmer DG. Hand grip function in patients with rheumatoid arthritis. Arch Phys Med Rehabil 1980;61(8):369-373.

28. Byrd R, Jenness ME. Effect of maximal grip strength and initial grip strength on contraction time and on areas under force-time curves during isometric contraction. Ergonomics 1982;25(5):387-392.

29. Smith GA, Nelson RC, Sadoff SJ, Sadoff AM. Assessing sincerity of effort in maximal grip strength tests. Am J Phys Med Rehabil 1989;68(2):73-80.

30. Yamaji S, Demura S, Nagasawa Y, Nakada M. The influence of different target values and measurement times on the decreasing force curve during sustained static gripping work. J Physiol Anthropol Appl Human Sci 2006;25(1):23-28.

31. Shechtman O, Sindhu BS, Davenport PW. Using the Force-Time Curve to Detect Maximal Grip Strength Effort. J Hand Ther 2007;20(1):37-47.

32. Bautmans I, Gorus E, Njemini R, Mets T. Handgrip performance in relation to self-perceived fatigue, physical functioning and circulating IL-6 in elderly persons without inflammation. BMC Geriatr 2007;7(5):1-8.

33. Young VL, Pin P, Kraemer BA, Gould RB, Nemergut L, Pellowski M. Fluctuation in grip strength and pinch strength among normal subjects. J Hand Surg 1989;14a(1):125-129.

34. Armstrong CA, Oldham JA. A comparison of dominant and non-dominant hand strengths. J Hand Surg 1999;24(4):421-415.

35. Anakwe RE, Huntley JS, Mceachan JE. Grip strength and forearm circumference in a healthy population. J Hand Surg 2007; 32(2):203-209.
36. Jürimäe T, Hurbob T, Jürimäe J. Relationship of handgrip strength with anthropometric and body composition variables in prepubertal children. HOMO J Comp Hum Biol 2009;60(3):225-238.

37. Visnapuu M, Jürimäe T. Handgrip strength and hand dimensions in young handball and basketball players. J Strength Cond Res 2007;21(3):923-929.

38. Vanderburgh PM, Mahar MT, Chou CH. Allometric. scaling of grip strength by body mass in college-age men and. women. Res Q Exerc Sport 1995;66(1):80-84.

39. Markovic G, Jaric S. Movement performance and body size: the relationship for different groups of tests. Eur J Appl Physiol 2004;92(1):139-149.

40. Pua Y. Allometric Analysis of Physical Performance Measures in Older Adults. Phys Ther 2006;86(9):1263-1270.

\section{Endereço para correspondência}

Jonathan Ache Dias

Laboratório de Instrumentação - LABIN

Centro de Ciências da Saúde e do Esporte - CEFID

Universidade do Estado de Santa Catarina - UDESC

Rua Pascoal Simone, 358. Bairro Coqueiros, CEP 88080-350 - Florianópolis, SC, Brasil

E-mail: jonathanache@gmail.com 